\title{
Association of Postoperative Complications with Hospital Costs and Length of Stay in a Tertiary Care Center
}

\author{
Nadia A. Khan, MD, MSc, ' Hude Quan, MD, PhD, ${ }^{2}$ Jennifer M. Bugar MD, ${ }^{3}$ \\ Jane B. Lemaire, MD, ${ }^{3}$ Rollin Brant, PhD, ${ }^{2}$ William A. Ghali, MD, MPH ${ }^{2,3}$ \\ 'Department of Medicine, University of British Columbia, BC, Canada; ${ }^{2}$ Department of Community Health Sciences, University of Calgary, \\ Calgary, AB, Canada; ${ }^{3}$ Department of Medicine, University of Calgary, Calgary, AB, Canada.
}

BACKGROUND: Postoperative complications are a significant source of morbidity and mortality. There are limited studies, however, assessing the impact of common postoperative complications on health care resource utilization.

OBJECTIVE: To assess the association of clinically important postoperative complications with total hospital costs and length of stay (LOS) in patients undergoing noncardiac surgery.

METHODS: We determined total hospital costs and LOS in all patients admitted to a single tertiary care center between July 1, 1996 and March 31, 1998 using a detailed administrative hospital discharge database. Total hospital costs and LOS were adjusted for preoperative and surgical characteristics.

RESULTS: Of 7,457 patients who underwent noncardiac surgery, $6.9 \%$ developed at least 1 of the postoperative complications. These complications increased hospital costs by $78 \%$ (95\% confidence interval [CI]: $68 \%$ to $90 \%$ ) and LOS by $114 \%$ (95\% CI: $100 \%$ to $130 \%$ ) after adjustment for patient preoperative and surgical characteristics. Postoperative pneumonia was the most common complication (3\%) and was associated with a $55 \%$ increase in hospital costs (95\% CI: $42 \%$ to $69 \%$ ) and an $89 \%$ increase in LOS (95\% CI: $70 \%$ to $109 \%)$

CONCLUSIONS: Postoperative complications consume considerable health care resources. Initiatives targeting prevention of these events could significantly reduce overall costs of care and improve patient quality of care.

KEY WORDS: perioperative; surgery; cost;complications DOI: $10.1111 /$ j.1525-1497.2006.00319.x J GEN INTERN MED 2006; 21:177-180.

A significant number of patients undergoing noncardiac surgery may experience a postoperative complication. Several studies ${ }^{1-3}$ reported rates of wound infection of $1.8 \%$ to $7 \%$, myocardial infarction (MI) $0.5 \%$ to $1 \%$, pneumonia $0.7 \%$ to $2.4 \%$, and stroke $<1 \%$ depending on the region studied, surgical procedure, and method of identifying complications. Meanwhile, studies that report health care costs and resource utilization associated with these complications are limited by focusing on single surgical procedures (especially coronary artery bypass graft surgery), ${ }^{4-7}$ examining only cardiac complications, ${ }^{8}$ using patient charges as a proxy for cost ${ }^{4}$ instead of using total (direct and indirect) hospital costs, or because costs are not adjusted for baseline conditions. ${ }^{9}$ To overcome

The authors have no conflicts of interest to declare for this paper.

Address correspondence and requests for reprints to Dr. Khan: Centre for Health Evaluation and Outcome Sciences, 620B-1081 Burrard Street, St. Paul's Hospital, Vancouver, BC, Canada V6N 1 Y6 (e-mail: nakhan @shaw.ca). these limitations, we studied the association of total hospital costs and length of stay (LOS) with multiple postoperative complications for a heterogeneous group of surgical procedures at a large tertiary care center.

\section{METHODS}

\section{Study Population}

The study population was derived from administrative hospital discharge data of an urban, 750-bed tertiary care center and teaching hospital in Calgary, Alberta, Canada, for patients discharged from hospital between July 1, 1996 and March 31, 1998. We included patients aged 16 or older admitted for elective or urgent noncardiac surgery. We excluded patients who were undergoing day procedures, had surgery cancelled, or who had no recorded procedure code $(2,151$ patients excluded in total).

\section{Data Source}

The administrative discharge abstract data coded by trained professional coders contain up to 16 diagnosis and 10 procedure codes of the International Classification of Diseases, Ninth Revision, Clinical Modification codes (ICD-9-CM), ${ }^{10}$ and itemized hospital costs. Total itemized costs, calculated based on the patient's Diagnosis-Related Group, represent estimated expenses accrued from direct and indirect patient care. Direct costs are based on all costs for providing patient care such as nursing staff, drugs, and other patient supplies. Indirect costs represent the cost of supporting the provision of patient care and include costs of administrative infrastructure, fixed facility costs, health records costs, etc. Physician salaries and billings are not included in hospital costs.

\section{Explanatory Variables}

To determine the independent association of postoperative complications on total cost and LOS, we adjusted for comorbid status as well as type of surgery (major vs minor). Comorbid status was defined using the Elixhauser list of 30 comorbid conditions. This list was validated for LOS and hospital costs as outcomes and has superior control of confounding compared with other comorbidity indices. ${ }^{11,12}$ Further, all comorbid conditions were identified by a diagnosis-type indicator that was recorded in the administrative data to flag

Manuscript received October 19, 2004

Initial editorial decision June 10, 2005

Final acceptance October 3, 2005 
conditions arising in-hospital ${ }^{13}$ to minimize misclassification of comorbidities as complications. Minor surgery was defined as a surgical procedure that involved brief anesthesia, limited tissue dissection, or anticipated short recovery period, i.e., $<2$ days. Examples of minor surgical procedures include hernia repair, mastectomy, arthroscopic and laparoscopic procedures. Examples of major surgery include: colectomy, thoracotomy, and lumbar laminectomy. Two independent reviewers classified over 1,000 surgical procedures in the database as minor or major. ${ }^{14}$ The percentage agreement between the 2 reviewers was $73 \%$, corresponding to a $\kappa$ score of 0.42 , indicative of moderate agreement. Any disagreements were resolved by consensus.

\section{Postoperative Complications}

To identify postoperative complications that were common and/or clinically important, we used ICD-9-CM diagnosis codes (codes beginning with 996-999) that are explicitly labeled as "complications" (cardiac complications, pneumonia, hemorrhage, infectious complication, stroke) as well as ICD9-CM codes for acute MI, acute respiratory failure, and deep vein thrombosis/pulmonary embolism. These codes have been used in other studies ${ }^{15,16}$ (see online Appendix 1).

\section{Statistical Analysis}

The logarithmic transformation of cost and LOS was used because of the positive skew in the distribution of these 2 variables. To estimate the independent association between complications and costs and LOS, we adjusted for baseline characteristics including age, gender, the Elixhauser comorbidity list, urgency of surgery (urgent vs nonurgent), and type of surgery (major vs minor) using linear regression modeling. Clinically and statistically relevant interaction terms were also included. The final model was assessed for the satisfaction of assumptions for multiple linear regression. We also evaluated for potential clustering at the surgical service level using a linear mixed effects modeling procedure. That analysis supported the findings from the simpler linear regression models that we present in the Results section.

The adjusted cost increase (i.e., the relative cost increase associated with the presence of a given complication vs the absence of any complications) was estimated by the percentage increase of the exponentiated $\beta$ coefficient. We determined the median cost with a given complication by multiplying the adjusted percent increase that was associated with a postoperative complication with the unadjusted geometric mean. The same method was used to assess increase in LOS.

\section{RESULTS}

There were 7,457 patients undergoing noncardiac surgical procedures during the 21-month study period. As discerned from Table 1 , the majority of patients in this study were female, with generally few comorbid conditions.

A significant proportion of patients experienced at least 1 postoperative complication (6.9\%) but the occurrence of more than 1 complication was infrequent. The most frequent complications were postoperative pneumonia (3\%), hemorrhage (1.8\%), postoperative infection (1.3\%), and cardiac complications (1.3\%).
Table 1. Patient Characteristics $(n=7,457)$

\begin{tabular}{|c|c|}
\hline Characteristic & \\
\hline \multicolumn{2}{|l|}{ Age (y) } \\
\hline Mean $\pm \mathrm{SD}$ & $50 \pm 17$ \\
\hline Range & 16 to 93 \\
\hline Female & $63 \%(4,696)$ \\
\hline Major surgery* & $62 \%(4,597)$ \\
\hline Urgent surgery & $41 \%(3,080)$ \\
\hline \multicolumn{2}{|l|}{ Surgery type } \\
\hline General & $31 \%(2,292)$ \\
\hline Neurosurgery & $19 \%(1,416)$ \\
\hline Obstetrics and Gynecology & $24 \%(1,780)$ \\
\hline Orthopedics & $15 \%(1,134)$ \\
\hline Other & $11 \%(835)$ \\
\hline \multicolumn{2}{|l|}{ Comorbidities } \\
\hline Diabetes mellitus & $4 \%(273)$ \\
\hline Chronic pulmonary disease & $10 \%(763)$ \\
\hline Neoplasia & $15 \%(1,094)$ \\
\hline Liver disease & $0.6 \%(45)$ \\
\hline Hypertension & $20 \%(1,457)$ \\
\hline Cerebrovascular disease & $2 \%(166)$ \\
\hline Congestive heart failure & $1 \%(67)$ \\
\hline Recent myocardial infarction & $3 \%(231)$ \\
\hline \multicolumn{2}{|l|}{ Postoperative complications } \\
\hline Cardiac $^{\dagger}$ & $1.3 \%(100)$ \\
\hline Pneumonia & $3.0 \%(220)$ \\
\hline Stroke & $0.1 \%(9)$ \\
\hline Hemorrhage $^{\ddagger}$ & $1.8 \%(137)$ \\
\hline Infection ${ }^{\S}$ & $1.3 \%(93)$ \\
\hline Acute myocardial infarction $\|$ & $0.1 \%(9)$ \\
\hline Acute respiratory failure $\|^{\|}$ & $0.4 \%(27)$ \\
\hline $\mathrm{DVT} / \mathrm{PE}^{\|}$ & $0.2 \%(16)$ \\
\hline At least one complication & $6.9 \%(517)$ \\
\hline Any two complications & $0.9 \%(67)$ \\
\hline Any three complications & $0.1 \%(6)$ \\
\hline Mortality & $0.2 \%(14)$ \\
\hline \multicolumn{2}{|l|}{ Cost in dollars ${ }^{* *}$} \\
\hline Median & 2,094 \\
\hline Range & 154 to 80,589 \\
\hline \multicolumn{2}{|l|}{ Length of hospital stay in days } \\
\hline Median & 3 \\
\hline Range & 1 to 116 \\
\hline
\end{tabular}

* Major surgery refers to any surgical procedure that is long in duration and involving a significant amount of tissue dissection or fluid shifts.

Cardiac complication is defined in the 996 series ICD-9-CM coding manual as cardiac arrest, cardiac insufficiency, cardiorespiratory failure, or heart failure during or resulting from a surgical procedure.

${ }^{\ddagger}$ Hemorrhage is defined in the 996 series ICD-9-CM coding manual as any hemorrhage of any site resulting from a surgical procedure.

${ }^{\S}$ Infection is defined in the 996 series ICD-9-CM coding manual as postoperative abscess or septicemia or infection due to an internal prosthetic device.

"These comorbid conditions are defined using ICD-9 codes not in the 996 series. $^{15}$

'Any complication refers to the occurrence of at least 1 of the following postoperative complications: cardiac, pneumonia, infection, stroke, $D V T / P E$, acute respiratory failure, MI, or hemorrhage.

**All dollar values are based on 1998 U.S. dollars.

ICD-9-CM, International Classification of Diseases, Ninth Revision, Clinical Modification codes; DVT/PE, deep vein thrombosis/pulmonary embolism.

In the multivariate analysis, after adjustment for age, gender, preoperative comorbid conditions, type of surgical procedure, urgency of surgery, the presence of a postoperative complication compared with having no complications was independently associated with increased total hospital costs (Table 2). Postoperative complications were also associated with increases in LOS except for acute MI. Postoperative stroke and infectious complications were associated with the largest 
Table 2. Increases in Total Hospital Cost and LOS for Postoperative Complications Before and After Adjustment for Patient Characteristics and Comorbid Conditions

\begin{tabular}{|c|c|c|c|c|c|c|}
\hline Complication & $\begin{array}{l}\text { Median LOS } \\
\quad(I Q R)\end{array}$ & $\begin{array}{l}\text { Unadjusted \% } \\
\text { Increase LOS } \\
(95 \% \mathrm{Cl})\end{array}$ & $\begin{array}{c}\text { Adjusted } \% \\
\text { Increase LOS* } \\
(95 \% \mathrm{Cl})\end{array}$ & $\begin{array}{l}\text { Median Cost } \\
\qquad(I Q R)^{\ddagger}\end{array}$ & $\begin{array}{l}\text { Unadjusted } \% \\
\text { Increase Cost } \\
(95 \% \mathrm{Cl})\end{array}$ & $\begin{array}{c}\text { Adjusted } \% \\
\text { Increase Cost } \\
(95 \% \mathrm{Cl})\end{array}$ \\
\hline Pneumonia & 7.0 (4.0 to 12.0 ) & 164 (142 to 188 ) & 75 (58 to 94$)$ & $3,930(2,511$ to 7,505$)$ & 100 (80 to 123$)$ & 47 (34 to 60$)$ \\
\hline Stroke & $11.0(9.0$ to 15.0$)$ & 297 (71 to 2,399$)$ & 98 (22 to 222 ) & $7,394(4,274$ to 10,054$)$ & 277 (100 to 608 ) & 112 (39 to 223 ) \\
\hline Hemorrhage & 7.0 (5.0 to 12.0 ) & 160 (137 to 186$)$ & 87 (64 to 94$)$ & $4,747(2,922$ to 6,616$)$ & 112 (92 to 133$)$ & $63(46$ to 81$)$ \\
\hline Infectious & $10.0(4.0$ to 16.9$)$ & 190 (139 to 253 ) & 122 (91 to 159$)$ & $4,747(2,788$ to 8,805$)$ & 126 (91 to 165$)$ & 79 (57 to 105$)$ \\
\hline Cardiac & 9.0 (6.0 to 14.5$)$ & 218 (177 to 264$)$ & 46 (24 to 72$)$ & $5,767(3,595$ to 9,637$)$ & 191 (152 to 234$)$ & 65 (43 to 90$)$ \\
\hline AMI & $20.9(2.0$ to 46$)$ & $286(16$ to 1,180$)$ & 3 (38 decr to 70 incr) & $11,309(6,524$ to 25,168$)$ & 372 (120 to 955$)$ & $50(0$ to 133$)$ \\
\hline $\begin{array}{l}\text { Acute respiratory } \\
\text { failure }\end{array}$ & $6.0(4.0$ to 12.9$)$ & 131 (63 to 225$)$ & 35 (2 to 79$)$ & $3,345(2,515$ to 8,509$)$ & $114(47$ to 211$)$ & $41(10$ to 80$)$ \\
\hline $\mathrm{DVT} / \mathrm{PE}$ & $8.0(7.0$ to 16.4$)$ & 199 (108 to 324$)$ & 103 (41 to 193$)$ & $6,551(4,287$ to 9,566$)$ & 194 (120 to 294 ) & 106 (50 to 183 ) \\
\hline Any complication ${ }^{\dagger}$ & 7.0 (4.0 to 11.9 ) & 165 (151 to 178 ) & $101(87$ to 115$)$ & $4,278(2,734$ to 6,656$)$ & 110 (99 to 122$)$ & 71 (61 to 82$)$ \\
\hline
\end{tabular}

*Adjusted increase in cost/LOS refers to cost/LOS associated with a complication compared with the cost/LOS without any complication.

Any complication refers to the occurrence of at least 1 of the following postoperative complications: cardiac, pneumonia, infection, stroke, hemorrhage, acute respiratory failure, acute myocardial infarction, or DVT/PE.

${ }^{\ddagger}$ All dollar values are based on 1998 U.S. dollars.

decr, decrease; incr, increase; LOS, length of stay; IQR, interquartile range; CI, confidence interval; AMI, acute myocardial infarction; DVT/PE, deep vein thrombosis/pulmonary embolism.

increases in cost and also in LOS. The presence of any complication was associated with a $110 \%$ increase in adjusted cost and $101 \%$ increase in LOS.

In addition, significant interactions between major type of surgery and pneumonia $(P<.01)$ and major type of surgery and postoperative cardiac complications $(P<.01)$ were observed. The interactions suggested that although these postoperative complications were associated with increased cost and LOS for both major and minor surgical procedures, the relative cost increase was greater for those undergoing minor surgeries.

\section{DISCUSSION}

We found that postoperative complications were relatively common, occurring in $6.9 \%$ of surgical patients in the present study. Each of the postoperative complications studied were associated with substantial increases in total hospital cost and LOS, even after adjusting for type of surgery, urgency of surgery, and preoperative patient comorbid conditions. The relative increase in cost for each of the complications ranged from $41 \%$ to $112 \%$. Parallel to the increases seen in costs, complications were associated with similar significant increases in LOS. Pulmonary complications occurred most often and were associated with a substantial increase in hospital cost and LOS. These increases in hospital costs were largely driven by prolonged hospital stay. This conclusion is based on the fact that although most complication types were associated with an increase in hospital cost independent of LOS, the magnitude of the cost increase was considerably lower than in an analysis that does not control for LOS.

These results are consistent with and extend findings from previous investigations of the impact of various perioperative explanatory factors on health care resource utilization in noncardiac surgery. ${ }^{8,12,17}$ Dimick et al. ${ }^{3}$ reported increases in hospital costs and LOS with several postoperative complications in a study of 1,008 surgical patients. However, because of the small number of events, they were only able to study a limited number of complications and could only adjust for a few comorbid conditions. Our study demonstrated, in a large patient population adjusted for a comprehensive list of comorbid conditions, that complications were associated with sub- stantial costs and prolonged LOS for multiple adverse complications.

The present study has several limitations. The first limitation is that as the costs and LOS results are from a single institution, they may not be representative of other hospitals. However, the complication rates in this study are similar to other reported complication rates from tertiary care centers. ${ }^{8,18,19}$ Second, administrative data might be incomplete and inaccurate in identifying complications. We reduced misclassification of comorbid conditions as complications by using the diagnosis-type indicator ${ }^{13}$ that identifies each comorbid condition as present at admission. Furthermore, Lawthers et al. ${ }^{16}$ reported that $73 \%$ of surgical complications identified by ICD- 9 codes were confirmed against medical record review. A third limitation is that the extreme imbalance of comorbidity profiles between patients with complications and those without might result in incomplete adjustment for potential confounding. While we could not use multivariable matched analyses proposed by some authors ${ }^{20}$ because of the low matching rate, we have attempted to reduce confounding by using a comprehensive list of comorbid conditions. ${ }^{11,12}$ The fourth limitation is that patients in this study underwent surgery between 1996 and 1998 and since that time, direct and indirect hospital costs may have increased. Also, costs in the single payer Canadian system are generally lower than quoted costs or charges from the American system. However, the overall conclusion that postoperative complications are independently associated with a significant increase in cost and LOS remains true, and is likely to apply to more recent Canadian data and to American data.

A final caveat is that we studied complications regardless of the occurrence of death. The relationship between death and costs/LOS is complicated, as death occurring early or late during the hospital admission would have differing effects on cost and LOS. In this study, the effect of death on resource use (cost and LOS) was, on average, modest, but this represents an averaged summary of effects that range anywhere from huge decreases in resource use (for early deaths) and huge increases in resource use (for later deaths).

In conclusion, patients with postoperative complications consume considerably more health care resources. Initiatives 
that target prevention of these events, even if costly to implement, would significantly improve quality of care and patient safety, with potential to also decrease the overall costs of care.

Dr. Khan is funded by Canadian Institutes of Health Research (CIHR) and Michael Smith Foundation for Health Research postdoctoral fellowship awards. Dr. Khan also received postdoctoral fellowship funding from the Alberta Heritage Foundation for Medical Research (AHFMR) during the analysis of this data. Dr. Ghali is funded by AHFMR as a Scholar and holds a Canada Research Chair. Dr. Hude Quan is funded by CIHR as New Investigator and AHFMR as a Population Health Investigator.

\section{REFERENCES}

1. Weingart SN, Iezzoni LI, Davis RB, et al. Use of administrative data to find substandard care: validation of the complications screening program. Med Care. 2000;38:796-806.

2. Fleischmann KE, Goldman L, Young B, Lee TH. Association between cardiac and noncardiac complications in patients undergoing noncardiac surgery: outcomes and effects on length of stay. Am J Med. 2003;115:515-20.

3. Dimick JB, Chen SL, Taheri PA, Henderson WG, Khuri SF, Campbell DA Jr. Hospital costs associated with surgical complications: a report from the private-sector National Surgical Quality Improvement Program. J Am Coll Surg. 2004;199:531-7.

4. Naglie G, Tansey C, Krahn MD, O'Rourke K, Detsky AS, Bolley H. Direct costs of coronary artery bypass grafting in patients aged 65 years or more and those under age 65. CMAJ. 1999;160:805-11.

5. Keon WJ, Menzies SC, Lay CM. Cost of coronary artery bypass surgery: a pilot study. Can J Surg. 1985;28:283-6.

6. Krueger H, Goncalves JL, Caruth FM, Hayden RI. Coronary artery bypass grafting: how much does it cost? CMAJ. 1992;146:63-8.

7. Lawrence VA, Dhanda R, Hilsenbeck SG, Page CP. Risk of pulmonary complications after elective abdominal surgery. Chest. 1996;1 10:744-50.

\section{Supplementary Material}

The following supplementary material is available for this article online:

\section{Appendix 1. List of complications of surgical care and} corresponding ICD-9-CM codes.

\section{Appendix 2. Full regression model for adjusted cost and} LOS.
8. Kalish RL, Daley J, Duncan CC, Davis RB, Coffman GA, Iezzoni LI. Costs of potential complications of care for major surgery patients. Am J Med Quality. 1995;10:48-54.

9. Naessens JM, Huschka TR. Distinguishing hospital complications of care from pre-existing conditions. Int J Qual Health Care. 2004; 16(suppl 1):i27-35.

10. U.S. Department of Health and Human Services. International Classification of Diseases, 9th revision (Clinical Modification). Washington, DC: Public Health Service, U.S. Department of Health and Human Services; 1998.

11. Elixhauser A, Steiner C, Harris DR, Coffey RM. Comorbidity measures for use with administrative data. Med Care. 1998;36:8-27.

12. Southern DA, Quan H, Ghali WA. Comparison of the Elixhauser and Charlson/Deyo methods of comorbidity measurement in administrative data. Med Care. 2004;42:355-60.

13. Guan H, Parsons GA, Ghali WA. Assessing accuracy of diagnosis-type indicators for flagging complications in administrative data. $\mathrm{J}$ Clin Epidemiol. 2004;57:366-72.

14. Bugar JM, Ghali WA, Lemaire JB, Guan H. Canadian Perioperative Research Network. Utilization of a preoperative assessment clinic in a tertiary care centre. Clin Invest Med. 2002;25:11-8.

15. McCarthy EP, Iezzoni LI, Davis RB, et al. Does clinical evidence support ICD-9-CM diagnosis coding of complications? Med Care. 2000; 38:868-76.

16. Lawthers AG, McCarthy EP, Davis RB, Peterson LE, Palmer RH, Iezzoni LI. Identification of in-hospital complications from claims data. Is it valid? Med Care. 2000;38:785-95.

17. Collins TC, Daley J, Henderson WH, Khuri SF. Risk factors for prolonged length of stay after major elective surgery. Ann Surg. 1999;230:251-9.

18. McAlister FA, Khan NA, Straus SE, et al. Accuracy of the preoperative assessment in predicting pulmonary risk after nonthoracic surgery. Am J Respir Crit Care Med. 2003;167:741-4.

19. Lee TH, Marcantonio ER, Mangione CM, et al. Derivation and prospective validation of a simple index for prediction of cardiac risk of major noncardiac surgery. Circulation. 1999;100:1043-9.

20. Zhan C, Miller MR. Excess length of stay, charges, and mortality attributable to medical injuries during hospitalization. JAMA. 2003; 290: 1868-74. 